\title{
Simulação de desempenho estrutural baseada na prototipagem rápida com impressão 3d
}

Structural performance simulation based on 3D printing for rapid prototyping

\author{
Andressa Carmo Pena Martinez \\ Universidade Federal de Viçosa, Brasil \\ andressamartinez@ufv.br \\ Douglas Lopes de Souza \\ Universidade Federal de Viçosa, Brasil \\ dglopes@gmail.com \\ Denise Mônaco dos Santos \\ Universidade Federal de Viçosa, Brasil \\ denise.monaco@ufv.br
}

\author{
Leonardo Gonçalves Pedroti \\ Universidade Federal de Viçosa, Brasil \\ Leonardo.pedroti@ufv.br
}

\author{
Marianna Auxiliadora Dias Martins \\ Universidade Federal de Viçosa, Brasil \\ Marianna.martins@ufv.br
}

\begin{abstract}
This paper presents part of the research on simulation of structural performance and aims to study the mechanical behavior of polymers, ABS and PLA in the form of thermoplastic filaments, commonly used in affordable 3D printers. It presents the preliminary results for the evaluation of the mechanical behavior of ABS and PLA in the light of ASTM E2954 and ASTM D790 standards, which establish test methods for axial compression and three-point flexure for plastic and polymer matrix.
\end{abstract}

Keywords: 3d printing; structural performance; rapid prototyping; computatuional simulation

\section{Introdução}

Segundo Hausler et al. (2017), técnicas e materiais de manufatura aditiva estão evoluindo rapidamente na última década. Aplicações na arquitetura, engenharia e construção estão ampliando o interesse da impressão 3D. No entanto, o custo do material, limitações da produção e dimensões dos modelos, bem como a performance estrutural (Hausler et al., 2017; Dritsas, 2017; Matson, 2016 e Crolla, 2014), ainda impedem o impacto significativo da aplicação da tecnologia de impressão 3D na produção de componentes de edificações.

A aplicação da manufatura aditiva, conhecida como impressão 3D - em arquitetura e engenharia tem sido limitada à criação de modelos físicos apenas com o propósito de representação, devido à relativamente baixa resistência das partes impressas em 3D. Neste sentido, esta pesquisa reúne pesquisadores dos campos de Arquitetura e Urbanismo e Engenharia Civil, com ênfase em projeto estrutural, e propõe-se a investigar a modelagem digital e a simulação de desempenho estrutural de componentes com geometrias complexas, produzidos a partir de impressoras 3D com filamentos aquecidos. O presente artigo apresenta os primeiros resultados dos ensaios destrutivos de corpos de prova, com o objetivo de conhecer o comportamento mecânico dos polímeros, ABS e PLA, sob a forma de filamentos termoplásticos, comumente utilizados em impressoras 3D de baixo custo.

Apesar do software de modelagem utilizado na pesquisa, o Rhinoceros e sua interação com o grasshopper constituir ambientes de simulação a partir de plugins de avaliação física como Karamba, Millipede, Kangoroo, Scan\&Solve, dentre outros (Hausler et al., 2017; Crolla, 2017 e Matson, 2016), ainda são necessários testes adequados para a compreensão do desempenho estrutural dos polímeros ABS e PLA. Esta fase da pesquisa visa lançar luz sobre este tema e auxiliar na validação de valores pré-existentes em plugins de simulação (como o ABS no Scan\&Solve) ou auxiliar na inserção e ponderação das propriedades de resistência dos materiais utilizados nas impressoras de baixo custo mais comuns nos laboratórios de fabricação digital. Esta pesquisa parte também da hipótese inicial que as limitações de qualidade, acabamento e velocidade de impressão, inerentes aos diferentes modelos de impressoras 3D, afetam o desempenho estrutural dos protótipos, e estes dados serão confrontados e ponderados ao final da investigação.

De fato, segundo Haeusler et al., 2017, em uma escala mais ampla, antes da introdução da manufatura aditiva em escala industrial ou no processo de construção, testes adequados de materiais, ensaios estruturais, códigos e normas precisam ser revistos. No entanto, as limitações atuais não reduzem o potencial para o desenvolvimento futuro da tecnologia (Naboni \& Paoletti, 2015).

\section{Objetivos}

Este artigo visa apresentar os resultados preliminares da avaliação do comportamento mecânico dos corpos de prova de material polimérico ABS e PLA, impressos por uma impressora 3D, submetidos a esforços de compressão axial e flexão, à luz das normas ASTM E2954 e ASTM D790 que 
estabelecem parâmetros para execução de ensaios de polímeros à compressão axial e à flexão em três pontos, respectivamente.

\section{Metodologia}

O procedimento metodológico da pesquisa completa possui quatro fases: (1) ensaios destrutivos dos polímeros $A B S$ e PLA para aferição do comportamento mecânico dos materiais; (2) desenvolvimento da modelagem e o sistema de geração formal de componentes impressos com geometrias complexas; (3) simulação e optimização na micro-escala de componentes, segundo metodologia de Haeusler et al. (2017) e Crolla (2017), utilizando plugins como Karamba, Millipede, Kangoroo e Scan\&Solve ${ }^{\mathrm{TM}}$. Nesta etapa, vislumbra-se a criação de modelos numéricos estruturais a partir dos ensaios destrutivos dos modelos físicos, que permitam a definição de novos algoritmos e componentes no grasshopper; (4) simulação e otimização da macro-escala do sistema estrutural, segundo metodologias de Haeusler et al. (2017), Crolla (2017), Matson (2016), utilizando plugins como Karamba.

Esse artigo trata especificamente da fase (1) da pesquisa, ensaios destrutivos de esforços de compressão axial e flexão, impressos prioritariamente no modelo de impressora 3D Cubex Duo, com área de impressão de $20 \mathrm{~cm}^{3}$. O método de modelagem e impressão inclui:

(a) estudo da otimização do rendimento da matéria-prima (filamentos termoplásticos ABS e PLA), e a relação entre o consumo e velocidade de impressão, dimensões dos modelos, massa e densidade do material;

b) qualidade de acabamento e precisão do modelo de acordo com as espessuras estabelecidas pela impressora $(0,1 \mathrm{~mm}$, $0,25 \mathrm{~mm}$ ou $0,5 \mathrm{~mm}$ );

(c) otimização de material e controle das malhas estruturais internas geradas e malhas de suporte (no caso de corpos de prova maciços);

(d) estratégias de finalização e pós-produção do modelo;

(e) geração de arquivos. STL e a análise qualitativa de modelos criados através dos softwares Rhinoceros e SketchUp;

(f) impressão de corpos de prova cilíndricos e prismáticos para ensaios destrutivos em dimensões e quantidades definidas pela equipe de análise estrutural;

(g) avaliação do comportamento mecânico dos corpos de prova, submetidos à esforços de compressão axial e flexão, à luz das normas ASTM E2954 e ASTM D790, que estabelecem parâmetros para execução de ensaios de polímeros à compressão axial e à flexão em três pontos, respectivamente;

(h) conclusões preliminares dos ensaios destrutivos.

\section{MATERIAIS E MÉTODOS}

\section{ETAPA DE IMPRESSÃO}

Como resultado da fase da impressão 3D, foram produzidos para os ensaios destrutivos 14 corpos de prova, sendo 7 formatados pelo software Cubify, padrão da impressora 3D Cubex Duo, com malha interna gerada automaticamente para otimização do consumo de matéria-prima, redução de custos e tempo de impressão. Durante o processo, percebeu-se a impossibilidade de impressão de corpos maciços no software Cubify, já que este definiu automaticamente uma malha interna estrutural, para a otimização do material. Por este motivo, adicionalmente foram formatados 7 corpos maciços, a partir do software KISSlicer, com o maior controle de camadas e suportes.

Cada conjunto com 7 corpos de prova, era caracterizado por 3 paralelípedos $\left(3 \times 2 \times 15 \mathrm{~cm}^{3}\right)$ e 4 cilindros nas dimensões de $2,5 \times 2,5 \times 5,0 \mathrm{~cm}^{3}$ e $5,0 \times 5,0 \times 10,0 \mathrm{~cm}^{3}$, impressos nos filamentos PLA e ABS. Os paralelepípedos foram impressos em seus eixos vertical e horizontal, para verificar a possível influência da orientação das fibras no desempenho estrutural. Os corpos de prova com malha estrutural interna foram impressos com espessuras de 0,1, 0,2 e 0,5 milímetros (seguindo o padrão da impressora Cubex Duo), densidade média e sem nenhum suporte, enquanto os maciços foram encapsulados por uma pele de suporte (figuras 1 e 2 ).

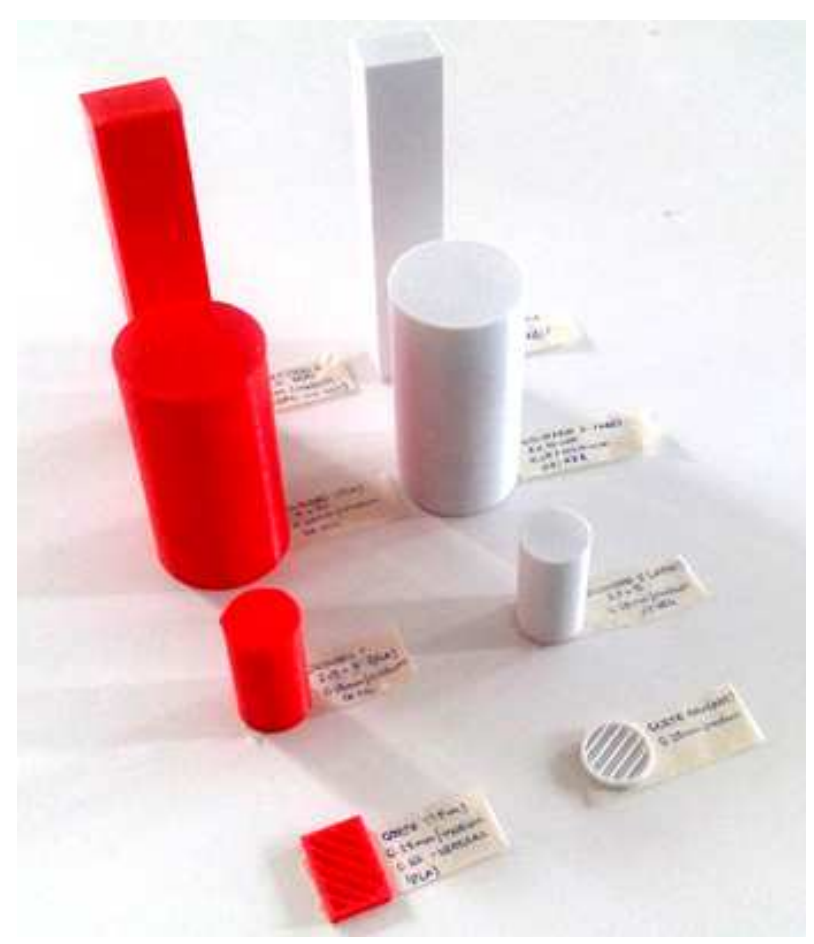

Figura 1: Os corpos de prova impressos verticalmente, com malha estrutural interna gerada pelo software Cubify, foram identificados por cores (laranja PLA e branco ABS) e respectivas configurações de impressão. 


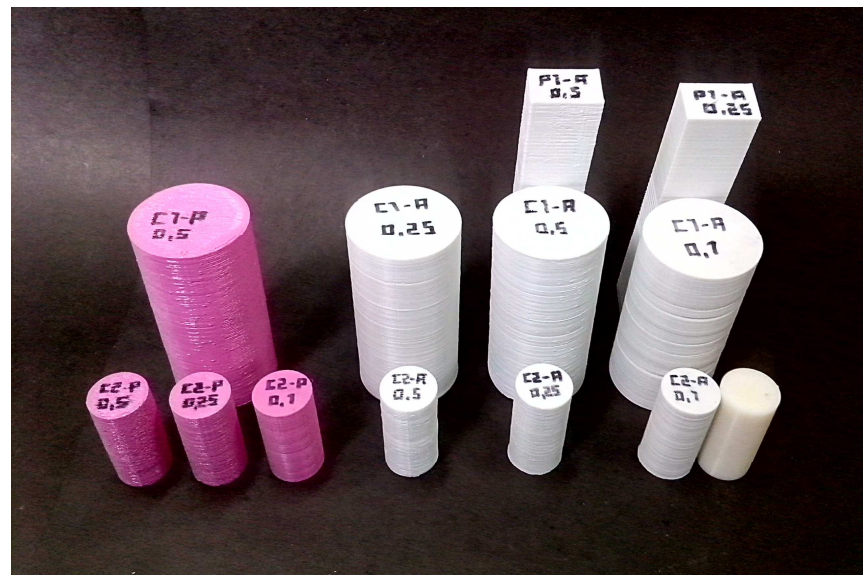

Figura 2: Os corpos de prova maciços, impressos verticalmente, foram identificados por cores (magenta PLA e branco ABS), e respectivas configurações de impressão.

Adicionalmente, foram impressos diversos cilindros com dimensões de $2,5 \times 2,5 \times 5,0 \mathrm{~cm}^{3}$ nas impressoras de baixo custo, RepRap e Prusa i3, e um único protótipo na impressora profissional Dimension 1200 com camadas de espessura de $0,025 \mathrm{~mm}$ de espessura, exclusivamente no material ABS. A figura 03 ilustra a diferença de qualidade de acabamento entre as impressoras de baixo custo (à esquerda) e as impressoras profissionais (à direita).

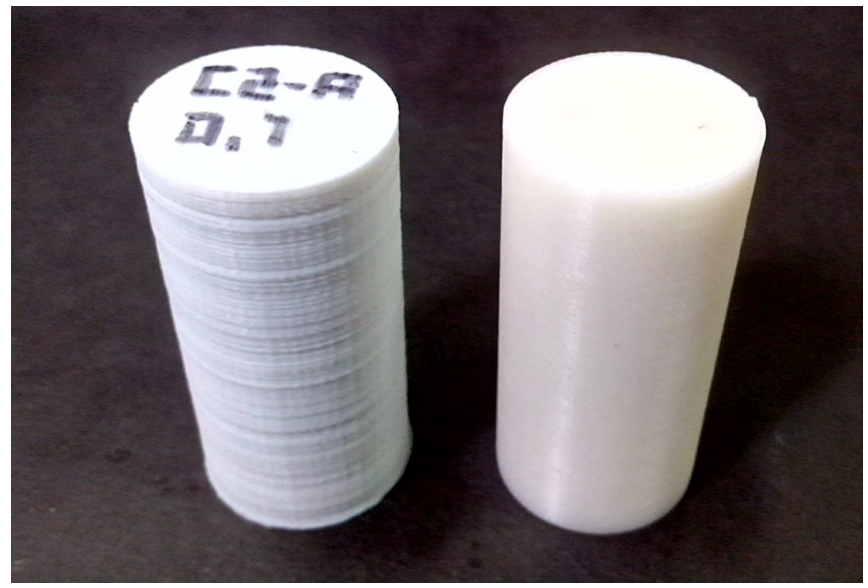

Figura 3: Comparação visual entre os cilindros em ABS. À esquerda, impresso por impressora de baixo custo com espessura de camada de $0,1 \mathrm{~mm}$ e à direita, com impressora profissional $e$ camadas de $0,025 \mathrm{~mm}$.

\section{PREPARAÇÃO ENSAIOS DESTRUTIVOS:}

As propriedades mecânicas dos materiais podem ser caracterizadas por vários métodos de ensaio, dentre os quais, o ensaio de compressão axial e o ensaio de flexão a três pontos. O primeiro refere-se a um esforço crescente de compressão distribuído de maneira uniforme ao longo da seção transversal do corpo de prova, de modo a provocar o seu encurtamento longitudinal; o segundo caracteriza-se por uma carga concentrada crescente aplicada no centro do vão de uma barra bi apoiada, com o objetivo de fletir a peça em torno do seu eixo de maior inércia.

\section{Identificação}

Os corpos de prova foram identificados de acordo com a procedência do material (ABS e PLA) e com a orientação de suas fibras internas, e suas dimensões (comprimento, largura e peso), aferidas com auxílio de régua e balança de precisão de $0,1 \mathrm{~g}$.

Em seguida os corpos de prova cilíndricos foram submetidos aos ensaios de compressão, enquanto os paralelepípedos à flexão:

\section{Ensaio de compressão axial}

Os corpos de prova cilíndricos foram centralizados na máquina de ensaios universal, utilizando dois extensômetros analógicos: um para a medição da carga e outro, para a medição do deslocamento da placa superior. A Figura 4 traz o esquema de montagem: A carga foi aplicada de maneira incremental e medida pelo extensômetro analógico de constante $\mathrm{K}=692 \mathrm{kgf} / \mathrm{mm}$, com valores de deformação da amostra obtidos em intervalos de medição de 0,05 $\mathrm{mm}$.

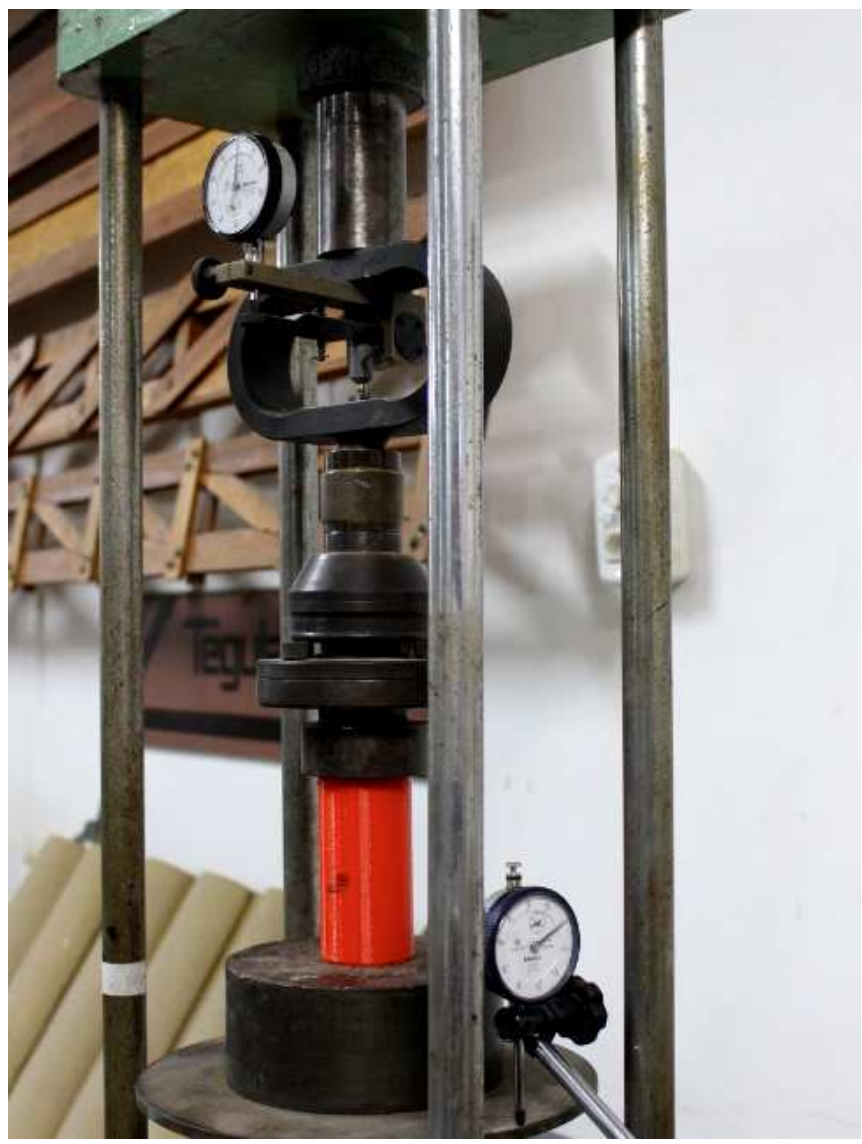

Figura 4: Esquema de montagem das amostras para o ensaio de compressão axial. 
A tensão de compressão para cada incremento foi calculada a partir da seguinte expressão: $\varepsilon=\frac{F}{A}(1)$, onde: $\mathrm{P}=$ Carga ; A = Área da seção inicial.

A deformação especifica é dada pela equação: $\varepsilon=\frac{\Delta L}{L i}(2)$, onde: $\Delta L=$ Variação de comprimento; $L i=$ Comprimento inicial.

\section{Ensaio de flexão em três pontos}

Os paralelepípedos foram marcados no centro vão e a $3 \mathrm{~cm}$ das duas extremidades, antes de posicionadas na máquina de ensaios universal (Figura 5). A carga foi aplicada de maneira incremental e medida com 0 extensômetro analógico de constante $\mathrm{K}=692 \mathrm{kgf} / \mathrm{mm}$.

A formula matemática para o cálculo do momento fletor máximo no centro do vão é dada em função da distância entre os apoios (L) e da carga aplicada (P): $M=\frac{P Z}{4}(\mathrm{~J})$

O momento de inércia para a seção retangular foi obtido através da relação: $l=\frac{b^{2}}{12}(4)$, onde: $b=$ dimensão da base da seção retangular, $h=$ dimensão da altura da seção retangular.

O modulo de resistência da seção transversal retangular, representado pela letra $\mathrm{W}$ pode ser obtido a partir da seguinte expressão: $W=\frac{l}{0.5 \hat{h}}(5)$

Por fim, a tensão de flexão na ruptura é dada pela equação: $\sigma=\frac{M}{W}(6)$

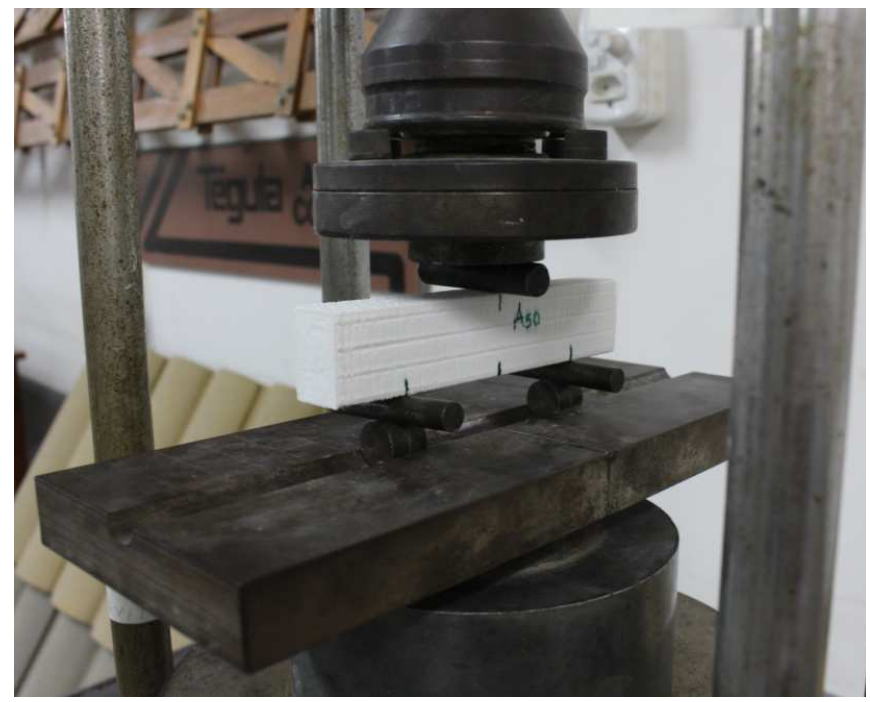

Figura 5: Preparação para o ensaio de flexão a três pontos.

\section{RESULTADOS \\ Etapa de impressão}

A tabela 1 identifica e compara os corpos de prova PLA e ABS finalizados e posteriormente testados, de acordo com as dimensões, massa e tempo de impressão.

Tabela 1: Comparação entre os corpos de prova maciços e com malha interna, no que se refere ao tempo de impressão e consumo de material.

\begin{tabular}{|c|c|c|c|c|c|c|}
\hline & \multirow[b]{2}{*}{$E$} & \multirow[b]{2}{*}{ DIMENSAO } & \multicolumn{2}{|c|}{$\operatorname{MASSA}(\mathrm{G})$} & \multicolumn{2}{|c|}{ TEMPO (HH:MM:SS) } \\
\hline & & & MACIÇO & MALHA & MACIÇO & MALHA \\
\hline \multirow{9}{*}{ ABS } & \multirow{3}{*}{0.50} & $\begin{array}{c}\text { CILINDRO } \\
(2.50 \times 5.00 \mathrm{~cm})\end{array}$ & 25,67 & 10,64 & $1: 03: 00$ & $0: 35: 00$ \\
\hline & & $\begin{array}{c}\text { CILINDRO } \\
(5.00 \times 10.00 \mathrm{~cm})\end{array}$ & 205,81 & 58,36 & 7:31:00 & $2: 18: 00$ \\
\hline & & $\begin{array}{l}\text { PARALELEPIPEDO } \\
(3.00 \times 2.00 \times \\
15.00 \mathrm{~cm})\end{array}$ & 94,59 & 38,41 & $4: 00: 00$ & $1: 49: 00$ \\
\hline & \multirow{3}{*}{0.25} & $\begin{array}{l}\text { CILINDRO } \\
(2.50 \times 5.00 \mathrm{~cm})\end{array}$ & 25,78 & 8,67 & $2: 06: 00$ & $2: 04: 00$ \\
\hline & & $\begin{array}{c}\text { CILINDRO } \\
(5.00 \times 10.00 \mathrm{~cm})\end{array}$ & 206,24 & 46,97 & $15: 02: 00$ & $3: 45: 00$ \\
\hline & & $\begin{array}{l}\text { PARALELEPIPEDO } \\
(3.00 \times 2.00 \mathrm{X} \\
15.00 \mathrm{~cm})\end{array}$ & 94,99 & 32,17 & $8: 00: 00$ & $2: 58: 00$ \\
\hline & \multirow{3}{*}{0.10} & $\begin{array}{c}\text { CILINDRO } \\
(2.50 \times 5.00 \mathrm{~cm})\end{array}$ & 25,83 & 10,2 & $5: 17: 00$ & $4: 51: 00$ \\
\hline & & $\begin{array}{l}\text { CILINDRO } \\
(5.00 \times 10.00 \mathrm{~cm})\end{array}$ & 206,50 & 60,49 & $37: 38: 00$ & $8: 52: 00$ \\
\hline & & $\begin{array}{l}\text { PARALELEPIPEDO } \\
(3.00 \times 2.00 \times \\
15.00 \mathrm{~cm})\end{array}$ & 95,214 & 39,15 & $20: 00: 00$ & 7:08:00 \\
\hline \multirow{9}{*}{ PLA } & \multirow{3}{*}{0.50} & $\begin{array}{l}\text { CILINDRO } \\
(2.50 \times 5.00 \mathrm{~cm})\end{array}$ & 28,12 & 11,02 & $1: 03: 00$ & $00: 32: 00$ \\
\hline & & $\begin{array}{l}\text { CILINDRO } \\
(5.00 \times 10.00 \mathrm{~cm})\end{array}$ & 225,41 & 54,72 & 7:31:00 & $1: 59: 00$ \\
\hline & & $\begin{array}{l}\text { PARALELEPIPEDO } \\
(3.00 \times 2.00 \times \\
15.00 \mathrm{~cm})\end{array}$ & 103,60 & 37,50 & $4: 00: 00$ & $1.31: 00$ \\
\hline & \multirow{3}{*}{0.25} & $\begin{array}{c}\text { CILINDRO } \\
(2.50 \times 5.00 \mathrm{~cm})\end{array}$ & 28,23 & 12,18 & $2: 06: 00$ & $1: 02: 00$ \\
\hline & & $\begin{array}{l}\text { CILINDRO } \\
(5.00 \times 10.00 \mathrm{~cm})\end{array}$ & 225,88 & 67,62 & $15: 02: 00$ & $4: 27: 00$ \\
\hline & & $\begin{array}{l}\text { PARALELEPIPEDO } \\
(3.00 \times 2.00 \times \\
15.00 \mathrm{~cm})\end{array}$ & 104,04 & 43,86 & $8: 00: 00$ & $3: 33: 00$ \\
\hline & \multirow{3}{*}{0.10} & $\begin{array}{c}\text { CILINDRO } \\
(2.50 \times 5.00 \mathrm{~cm})\end{array}$ & 28,29 & 13,05 & 5:17:00 & $1.58: 00$ \\
\hline & & $\begin{array}{c}\text { CILINDRO } \\
(5.00 \times 10.00 \mathrm{~cm})\end{array}$ & 226,17 & 71,90 & $37: 38: 00$ & $8: 42: 00$ \\
\hline & & $\begin{array}{l}\text { PARALELEPIPEDO } \\
(3.00 \times 2.00 \mathrm{x} \\
15.00 \mathrm{~cm})\end{array}$ & 104,28 & 47,22 & $20: 00: 00$ & $7: 04: 00$ \\
\hline
\end{tabular}

As limitações das impressoras de baixo custo ainda são entraves para assegurar um rígido controle de resistência e uniformidade da qualidade dos modelos. Abaixo descrevemos algumas limitações nas etapas de préimpressão, impressão e pós-produção do modelo:

Etapa de pré-impressão: A variação de umidade do ambiente ocasiona pequenas variações dimensionais nos filamentos, decorrentes de fenômenos de retração e expansão, ainda no cartucho. Essa variabilidade foi ainda mais significativa no PLA, o que ocasionou maior facilidade 
de rompimento dos fios durante a impressão, gerando falhas de fluxo do material.

Etapa de impressão: Peças maciças exigem maior atenção na configuração de parâmetros, sobretudo nas de menores dimensões. Os primeiros corpos de prova maciços apresentaram deformações causadas pelo extravasamento do preenchimento interno (figura 6), interferindo diretamente nos resultados de resistência. A redução da espessura da camada de preenchimento interno, e inversamente, o espessamento da parede externa, foram essenciais para que os corpos de prova apresentassem um melhor acabamento. Além disso, ajustes constantes de temperatura, altura do bico e velocidade, contribuíram com a melhoria da série de corpos de prova.

No que refere à integridade das camadas, a principal causa de descolamento é a variação de temperatura ou correntes de ar no ambiente: número de pessoas, fluxo de ar condicionado, amplitude térmica durante um único dia e temperatura noturna baixa, principalmente no caso das impressões que atingiram 30 a 40 horas. Para minimizar o problema, a impressora Cubex Duo foi vedada lateralmente para evitar o fluxo de ar transversal à mesa, além da modelagem de uma casca externa ao modelo, para auxiliar a adesão das camadas. Objetos impressos sem a casca de proteção apresentaram problemas de descolamento da mesa, além da separação de camadas (figura 6).

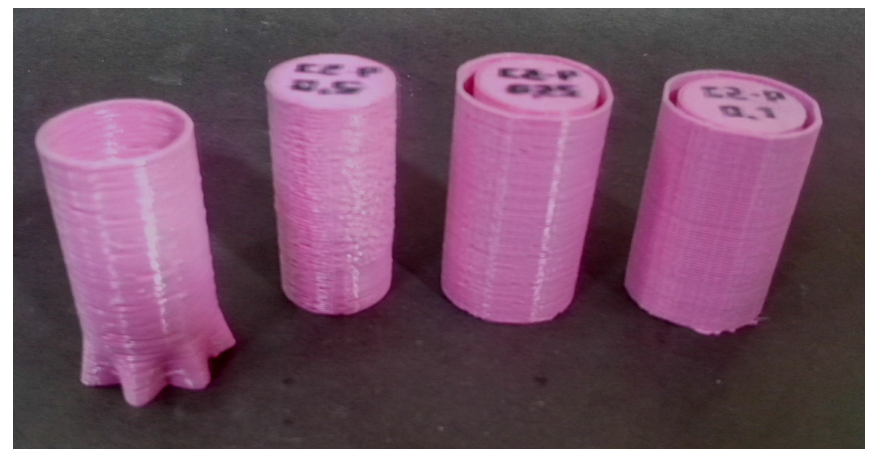

Figura 6: à esquerda, a camada indica extravasamento do material; os corpos de prova à direita possuem a casca de proteção criada para minimizar os problemas de impressão, decorrentes do descolamento das camadas.

Em impressões de longa duração (40 horas), as variações térmicas do ambiente são mais significativas, e a baixa velocidade de impressão diminui a manutenção de temperatura de extrusão, causando descolamento das camadas. Nestes casos, foi possível ouvir estalos decorrentes do desprendimento do material. No entanto, a casca de proteção, apesar de benéfica, impedia a visualização destas falhas durante o processo de impressão.

Etapa de pós-produção: Se durante a etapa de préprodução, o PLA sofreu maior variação dimensional decorrente de umidade e temperatura do ambiente, na fase de pós-produção, foi o ABS que apresentou menor durabilidade de integridade das camadas ao longo do tempo. A figura 7 ilustra como as falhas de impressão no $A B S$ aumentam no decorrer de poucos dias, principalmente nos corpos de prova com altura superior a $10 \mathrm{~cm}$ e longo tempo de impressão. Nos ensaios de resistência do material (figura 8), essas falhas nas camadas também são constatadas, mesmo em corpos de prova que possuíam visualmente, uma aparente integridade preservada.

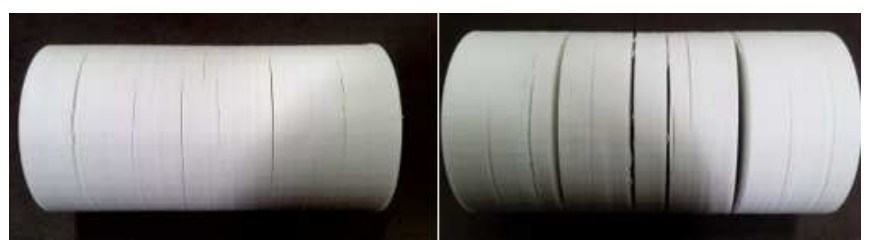

Figura 7: descolamento das camadas após o fim da impressão, em poucas horas, e acentuada ao longo de alguns dias.

Em geral, os maiores problemas durante a fase de impressão e pós-produção foram constatados nos corpos de prova maciços, decorrentes do maior tempo de impressão. A geração da malha interna automática, além de otimizar o consumo de material, reduz trincas, desprendimento de camadas e outras falhas, e prolongam a vida útil do objeto

\section{Etapa de ensaios destrutivos}

Para este artigo, foram ensaiados preliminarmente apenas os corpos de prova com malha estrutural interna, uma vez que a redução do tempo de impressão, consumo de material e, consequentemente, menores falhas durante o período de impressão e pós-produção aumentam a viabilidade de sua produção em escala. Os ensaios com os objetos maciços ainda estão em andamento.
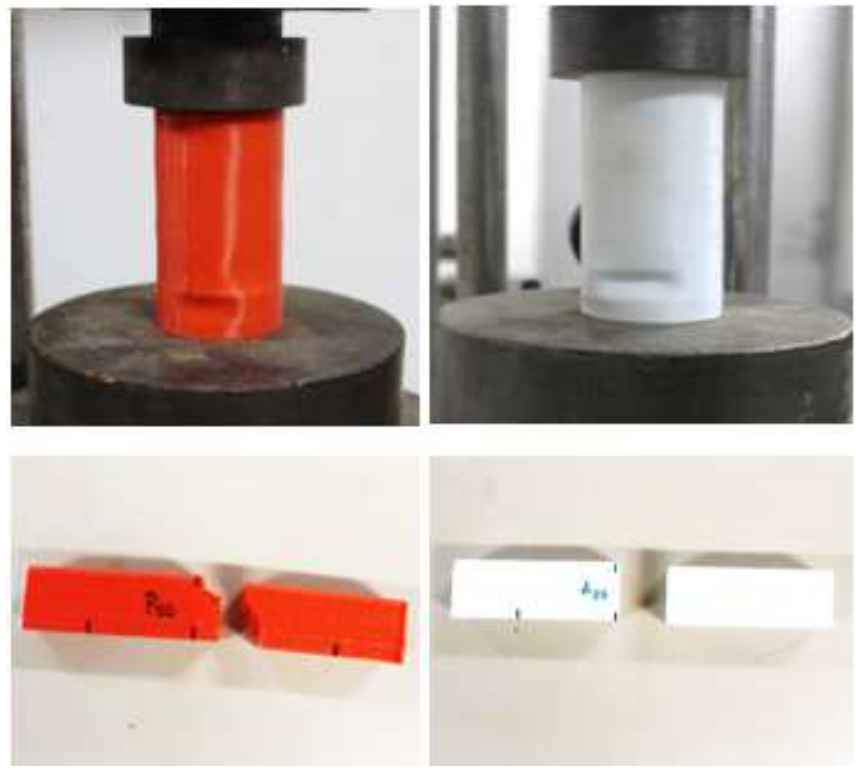

Figura 8: Amostras dos dois materiais rompidos à compressão axial e à flexão. $A$ amostra de $A B S$, abaixo à direita, indica ruptura devido a problemas de impressão (em uma única camada de filamento), sem relação com a resistência do material. 


\section{Ensaio de compressão axial}

Tanto nas amostras de PLA quanto nas de ABS, submetidas à compressão axial, os gráficos de tensão $\mathrm{x}$ deformação traduzem o comportamento frágil de ambos os materiais: falha sem deformação plástica visível. Ainda é possível observar uma variação maior entre os valores de módulo de elasticidade para as amostras de PLA. Para as amostras de $A B S$, a gráfica tensão $x$ deformação apresentam inclinações semelhantes para a mesma densidade de fibras.

Os gráficos 1,2 e 3 ilustram os resultados de tensão $x$ deformação para cada amostra ensaiada, e estão dispostos de acordo com a densidade de fibras do material (espessura das camadas).

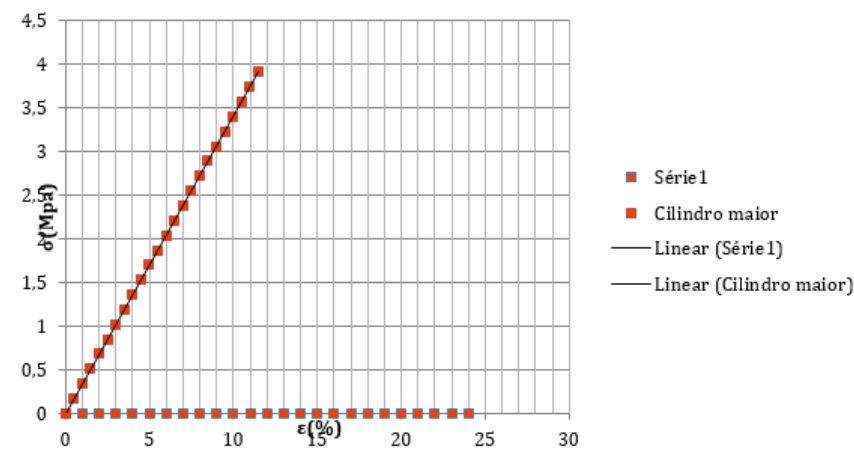

Gráfico 1: Curva tensão de deformação para as amostras de PLA (P50).

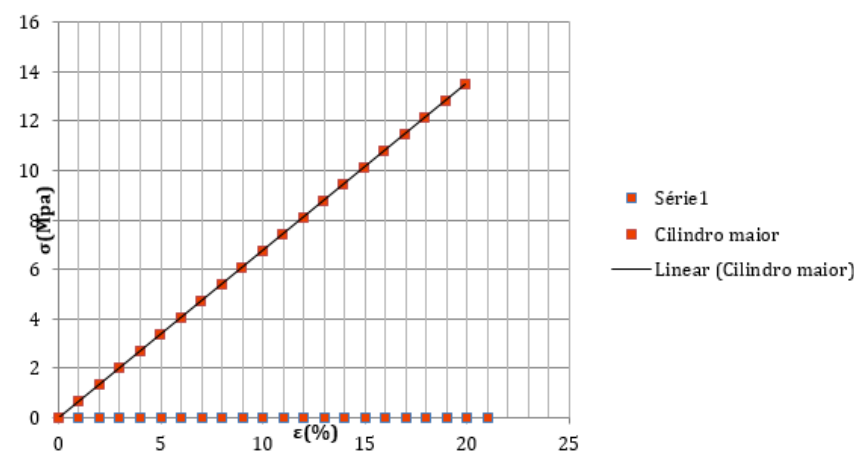

Gráfico 2: Curva tensão de deformação para as amostras de ABS (A25).

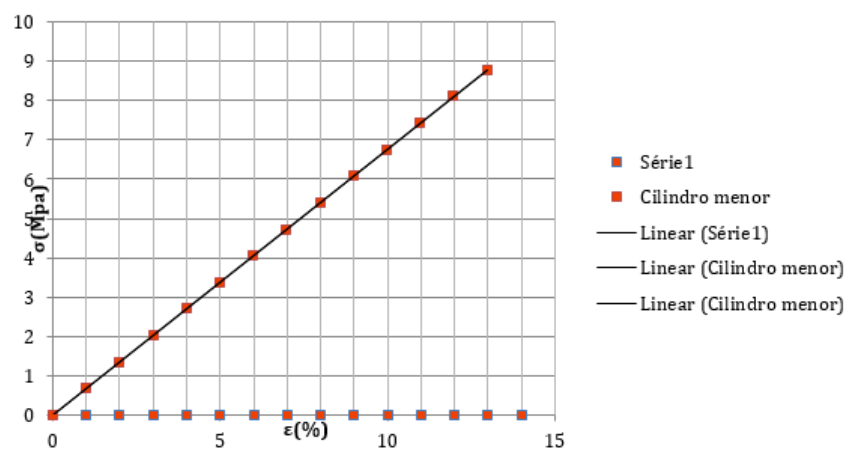

Gráfico 3: Curva tensão de deformação para as amostras de ABS (A50).

O modulo de elasticidade do material foi obtido a partir das médias da inclinação dos gráficos tensão x deformação: Para o PLA, E = 52,3 Mpa / Para o ABS, E = 65,42 Mpa

\section{Ensaio de flexão em três pontos}

Com relação aos ensaios de flexão, o maior momento cortante e momento fletor ocorrem no centro do vão. O carregamento produz esforços de compressão normal nas fibras superiores e tração nas inferiores. A disposição das fibras internas no material potencializa um comportamento anisotrópico, que confere diferentes propriedades de resistência à tração e à compressão. É possível notar nas amostras de plástico uma combinação de efeitos ao longo da seção do centro do vão: nas fibras inferiores prevalecem as tensões normais de tração, e nas superiores as tensões de cisalhamento, responsáveis pela fratura inclinada, no ponto de maior fragilidade do material. Para as amostras de ABS, a ruptura ocorre na junção entre os filetes da impressão, provavelmente ocasionadas por falhas de impressão.

A tabela 2 apresenta os resultados para os ensaios de flexão e as diversas propriedades de resistência de ambos os materiais, identificados pela letra P para PLA e A para ABS, seguido da espessura da camada. Segundo os testes, o PLA possui índices de resistência muito superiores ao ABS, no que se refere ao momento fletor máximo, tensão de flexão de ruptura, esforço cortante e tensão de cisalhamento. O ABS possui desempenho superior no módulo de elasticidade, e índices semelhantes para o momento de inércia e módulo de resistência. Também é notório que quanto menor a espessura das camadas, maior é o desempenho estrutural do sólido.

Tabela 2: Resultados para os ensaios de flexão. Onde, $\mathrm{CP}=$ corpo de prova, $\mathrm{P}=$ Carga, $\mathrm{I}=$ momento de inercia, $\mathrm{M}=$ Momento fletor máximo, $\mathrm{W}=$ módulo de resistência, $\sigma=$ tensão de flexão de ruptura, $\mathrm{Q}=$ carga variável, $\mathrm{V}=$ esforço cortante, $\tau=$ tensão tangencial ou de cisalhamento.

\begin{tabular}{cccccccccc}
\hline $\mathrm{P}$ & $\begin{array}{c}\mathrm{P} \\
(\mathrm{kN})\end{array}$ & $\begin{array}{c}\text { Área } \\
\left(\mathrm{cm}^{2}\right)\end{array}$ & $\begin{array}{c}\mathrm{I} \\
\left(\mathrm{cm}^{4}\right)\end{array}$ & $M f_{\text {máx }}(\mathrm{kN} \times \mathrm{cm})$ & $\underline{\underline{\mathrm{W}}\left(\mathrm{cm}^{3}\right)}$ & $\sigma(\mathrm{Mpa})$ & $\underline{\underline{\mathrm{Q}}\left(\mathrm{cm}^{3}\right)}$ & $\mathrm{V}(\mathrm{kN})$ & $\mathrm{T}(\mathrm{Mpa})$ \\
\hline $\mathrm{P} 25$ & 2.08 & 6.36 & 5.05 & 4.62 & 3.27 & 13.85 & 4.91 & 1.04 & 4.80 \\
\hline $\mathrm{P} 50$ & 1.73 & 6.19 & 4.74 & 3.89 & 3.13 & 12.22 & 4.69 & 0.87 & 4.12 \\
\hline $\mathrm{A} 25$ & 0.76 & 6.28 & 4.92 & 1.69 & 3.21 & 5.18 & 4.82 & 0.38 & 1.78 \\
\hline $\mathrm{A} 50$ & 0.69 & 6.62 & 5.39 & 1.54 & 3.45 & 4.38 & 5.17 & 0.35 & 1.54 \\
\hline
\end{tabular}

\section{Discussões futuras}

O trabalho apresentado constitui ainda uma investigação em processo e, portanto, requer outros ensaios, inclusive dos corpos de prova maciços. Para um estudo estatístico mais relevante, serão feitos também testes com uma amostragem maior.

Pretendemos também utilizar outros métodos normativos internacionais para testes mecânicos de plásticos e polímeros (ASTM), já utilizados em alguns plugins de simulação no Rhinoceros e Grasshopper, visando a 
comparação entre a simulação computacional e os ensaios físicos (fase 03 da pesquisa). Nesta etapa, vislumbra-se a criação de modelos numéricos estruturais a partir dos resultados dos ensaios destrutivos dos modelos físicos, que permitam a definição de novos algoritmos e componentes no Grasshopper.

A pesquisa também está em fase de exploração de corpos de prova produzidos em outros modelos de impressoras de baixo custo. Os resultados preliminares já indicam que a qualidade de acabamento dos objetos, relacionados também à menor espessura de camadas de filamento, contribui para a maior resistência dos materiais. Neste sentido, espera-se também que os testes destrutivos dos corpos de prova da impressora profissional Dimension 1200, não discutidos neste artigo, apontem para a viabilidade de uso da impressão 3D com filamentos termoplásticos, em maior escala e com relativo desempenho estrutural na produção de pequenos componentes construtivos.

\section{Agradecimentos}

Ao CNPq, financiador da bolsa de iniciação científica vinculada a este projeto de pesquisa.

\section{Referências}

American Society for Testing and Materials. (2002) ASTM D790: Standard Test Methods for Flexural Properties of Unreinforced and Reinforced Plastics and Electrical Insulating Materials. West Conshohocken, Pennsylvania, United States.

American Society for Testing and Materials. (2002) ASTM E2954: Standard Test Method for Axial Compression Test of Reinforced Plastic and Polymer Matrix Composite Vertical Members. West Conshohocken, Pennsylvania, United States.

Block, P., Lachauer, L., \& Rippmann, M. (2010). Validating Thrust Network Analysis using 3D-printed structural models. Proceedings of the International Association for Shell and Spatial Structures Symposium, Shangai, China.

Crolla, K., Williams, N. (2014). Smart Nodes: A System for variable structural frames with 3D Metal-Printed Nodes. Proceedings of the $34^{\text {rd }}$ International Conference of the Association for Computer-Aided in Architecture (ACADIA 14), 311-316.

Crolla, K., Williams, N., Muehlbauer, M., \& Burry, J. (2017). Smartnodes Pavilion. Proceedings of the $22^{\text {nd }}$ International Conference of the Association for Computer-Aided Architectural Design Research in Asia (CAADRIA), 467-477.

Dritsas, S., Chen, L., \& Sass, L. (2017). Small 3D Printers: Large Scale Artifacts. Proceedings of the $22^{\text {nd }}$ International Conference of the Association for Computer-Aided Architectural Design Research in Asia (CAADRIA), 821-831.

Haeusler, M. H., Muehlbauer, M., Bohnenberger, S., \& Burry, J. (2017). Furniture Design Using Custom-Optimised Structural Nodes. Proceedings of the $22^{\text {nd }}$ International Conference of the Association for Computer-Aided Architectural Design Research in Asia (CAADRIA), 841-851.

Huang, H.H., Wong, B.L, \& Chou, Y.C. (2016) Design and properties of 3D-printed chiral auxetic metamaterials by reconfigurable connections. Physica Status Solidi B 253, n.8, 1557-1564.

Matson, C. W., Sweet, K. (2016). Simplified for Resilience: A parametric investigation into a bespoke joint system for bamboo. Proceedings of the XX Congreso de la Sociedad Iberoamericana de Gráfica Digital (SIGRADI 16), 284- 289.
Naboni, R. and Paoletti, I. (2015), Additive Manufacturing, in R.P. Naboni (ed.), Advanced Customization in Architectural Design and Construction, Springer.

Peters, B. (2016). Additive formwork: 3D Printed Flexible Formwork. Proceedings of the $34^{\text {rd }}$ International Conference of the Association for Computer-Aided in Architecture (ACADIA 14), 517- 522.

Raspall, F., Banõn, C. (2016). VMesh: How to print Architecture? Proceedings of the XX Congreso de la Sociedad lberoamericana de Gráfica Digital (SIGRADI 16), 394-398.

Wasilkoski, C. M. (2006). Comportamento mecânico de materiais poliméricos. 68 f. Tese (Doutorado) - Curso de Engenharia dos Materiais, Universidade Federal do Paraná. 